\title{
An Optimal Algorithm for Energy Recovery of Hydraulic Electromagnetic Energy-Regenerative Shock Absorber
}

\author{
Zhigang Fang*, Xuexun Guo, Lin Xu and Han Zhang \\ Hubei Key Laboratory of Advanced Technology of Automotive Components, Wuhan University of Technology, Wuhan 430070, China
}

Received: 1 Mar. 2013, Revised: 27 Jun. 2013, Accepted: 28 Jun. 2013

Published online: 1 Nov. 2013

\begin{abstract}
This paper presents a new kind of shock absorber, hydraulic electromagnetic energy-regenerative shock absorber (HESA), which can recover energy from vibration of vehicles. The road excitation frequency, load resistance and damping ratio are found to greatly affect the energy recovery of HESA. Based on a quarter-car model, the optimal load resistance and damping ratio for maximizing the energy-recyclable power are discussed. The results indicate that for any excitation frequency, the energy-recyclable power first increases then decreases with the load resistance increase, and the optimal load resistance is changeable as the excitation frequency changes. Moreover, the energy-recyclable power is more sensitive to excitation frequency than to damping ratio, and the ideal excitation frequency for energy recovery is around the wheel resonant frequency. The exploration of active control of HESA confirms that the damping force varies with the load current magnitude, and the active control can be realized by appropriate matching.
\end{abstract}

Keywords: HESA, Optimal Algorithm, energy-recyclable power, optimal load resistance, optimal damping ratio.

\section{Introduction}

Energy harvest from the suspension has been studied for many years, and there are two common types of energy recovery shock absorbers: linear design and rotary design. Okada and Harada [1] proposed an electromagnetic energy regeneration system using a linear motor as an actuator, and the project was confirmed by a simple experimental apparatus. Suda [2] developed a hybrid suspension system, in which a linear DC generator was used to harvest energy from vibration. However, the linear motor is generally very expensive, and its efficiency was much lower than the rotary one's as Gupta reported [3]. And thus rotary design has attracted more and more attention due to its better application potential. Suda [4] developed an energy recovery shock absorber using DC motor and ball screw mechanism, and the performance of the trial electromagnetic damper was examined through numerical simulation and road test by passenger car equipped with the proposed damper. Yu [5] also built their energy recovery shock absorber using the same method, and the feasibility of vibration energy regeneration was confirmed by the bench test while the damping performance was poor for high-frequency excitation. The major obstacle to the application of the program is that, the motor changes rotation direction twice in each vibration cycle and this phenomenon seriously affects the high frequency response of the system, as well as the energy recovery efficiency. Zuo [6] modeled and tested a regenerative shock absorber based on rack and pinion mechanism, and the program was proved to be feasible by both simulation and test results. This design made great progress by adding a mechanical rectifier mechanism, and the motor would not change its rotation direction with the help of mechanical rectifier mechanism. However, this program has its drawbacks. Firstly, the frequency-limit of the mechanical rectifier mechanism needs to be concerned because the vehicle was often subjected to high-frequency excitation. Secondly, the backlash would seriously affects the reliability and durability of the system, as gear cracks even teeth broken may happen due to serious road impact.

In addition, the active control of suspension based on energy-regenerative shock absorber is also a hot topic. Suda [7] developed the active control electromagnetic suspension, and demonstrated the vibration isolation and regeneration on the vibration tests. From simulation, Huang and $\mathrm{Yu}$ [8] concluded that electromagnetic active suspension based on model predictive controller could obtain better ride comfort than linear optimal control could. Recently, the authors developed another rotary

\footnotetext{
* Corresponding author e-mail: zuoleng136@163.com
} 
design-hydraulic transmission [9,10,11], which based on hydraulic rectifier mechanism, hydraulic motor and generator. Compared with mechanical program, hydraulic rectifier mechanism has higher efficiency and hydraulic transmission program has better reliability and durability although the program has its shortcomings. In this paper, the working principle of the energy recovery program and its test bench are described briefly, and then the optimal energy recovery of HESA is analyzed amply based on the discussion. Furthermore, the active control exploration is also carried out in order to confirm the feasibility of HESA.

Prior to this study, it's also important to clarify how much energy is dissipated by the shock absorber when the car is being driven. Segel [12] analyzed the suspension system and found that a vehicle with four shock absorbers would dissipate about 200 watts with a speed of 13.4 $\mathrm{m} / \mathrm{s}$. Browne [13] tested the energy dissipated by four shock absorbers of a vehicle and found that the energy-dissipated power was about 40-60 watts. Zuo [14] proposed an energy dissipation formula and calculated the suspension system would dissipate 100-400 watts in B-C class road with a speed of $100 \mathrm{~km} / \mathrm{h}$.

\section{The design of HESA}

\subsection{The schematic of HESA}

The energy-regenerative program and its schematic are shown in Figure 1. It can be seen that HESA is composed of hydraulic cylinder, hydraulic rectifier, accumulators, hydraulic motor, generator, pipelines and so on. Its working process is as follows: the piston of the hydraulic cylinder is driven to reciprocate under external stimulus, the oil flows into and out of the hydraulic rectifier from the specified export in the compression stroke or extension stroke, and then the oil flows through the accumulator for weakening fluctuations, which drives the hydraulic motor to generate electricity. The electric energy can charge a battery or supply to the vehicle directly [11].

From Figure 1, it is also noticed that there are two accumulators fixed on both sides of the hydraulic motor. Accumulator 1 is used to stabilize the flow to improve the working efficiency of the hydraulic motor, and the function of accumulator 2 is to prevent high-frequency distortion of the shock absorber, which consists with the principle that filling the gas chamber of the twin-tube shock absorber with suitable-pressure gas. This design is helpful to make this scheme more practicable.

\subsection{Trial-manufacture of HESA}

The exploded view of our HESA model is shown in Figure 2. Accumulator 2, hydraulic rectifier and hydraulic

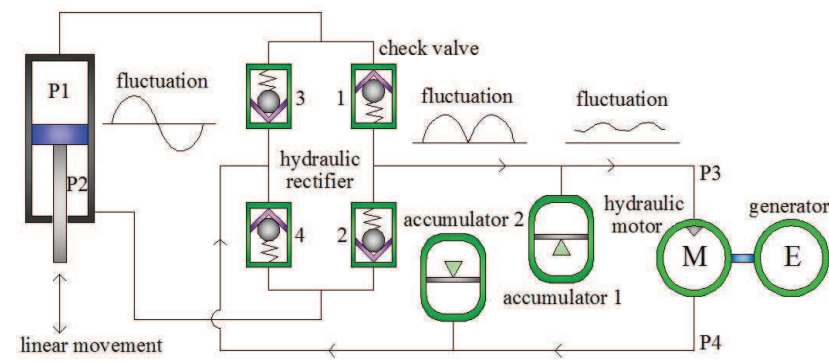

Fig. 1: The energy-regenerative program and schematic of HESA.

cylinder are integrated into the HESA, and the prototype of HESA is tentatively manufactured accordingly. And the test bench shown in Figure 3 is also built in accordance with the standard of QC/T 545-1999 [15]. During the test, necessary measures should be taken to ensure the obtained data reasonable, since oil leaking from connecting pipes or components, and air or entrapped gas in hydraulic circuit may affect the results of the test.

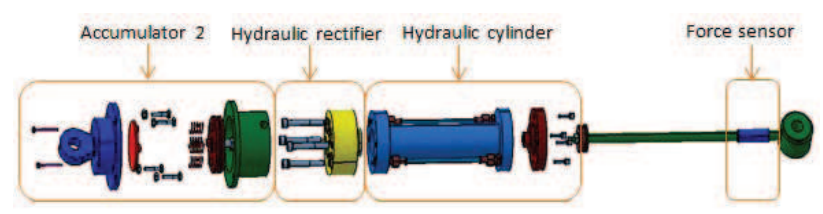

Fig. 2: Exploded view of HESA.

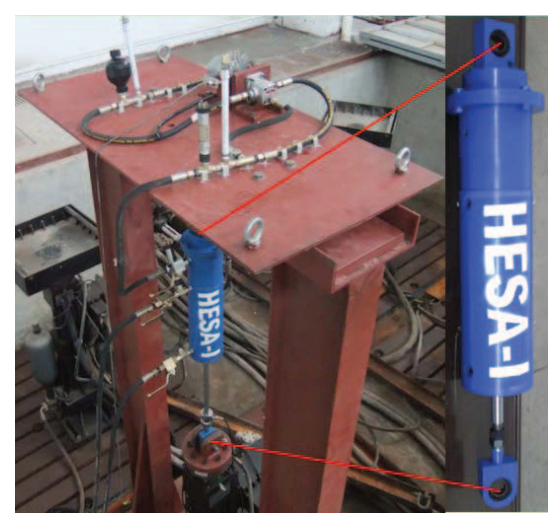

Fig. 3: Digital image for the test bench of HESA prototype. 


\section{Optimal energy recovery analysis of HESA}

The goal of optimal analysis of HESA is to recover energy as much as possible. For example, it should be installed in the rear suspension of commercial vehicles when the requirements of ride are not so serious. Besides that, if the real road excitation is random in frequency and amplitude, it would be hard to clarify the influence of frequency and amplitude on the energy recovery, so it is assumed in this section that the tire is subjected to harmonic excitations. Under this assumption, the recyclable energy is analyzed and the optimal conditions are derived based on the analysis.

\subsection{Harmonic Force Excitation}

Assuming the tire is subjected to force excitation, the governing equations of the vibration energy recovery system can be written as follows:

$$
m_{2} a_{2}+c_{e q}\left(v_{2}-v_{1}\right)+k_{2}\left(x_{2}-x_{1}\right)=0
$$

and

$$
\begin{gathered}
m_{1} a_{1}+c_{e q}\left(v_{1}-v_{2}\right)+k_{2}\left(x_{1}-x_{2}\right) \\
+k_{1} x_{1}=F \sin (\omega t)
\end{gathered}
$$

where $m_{1}$ and $m_{2}$ are the wheel mass and $1 / 4$ vehicle body mass respectively, $k_{1}$ and $k_{2}$ are the tire stiffness and suspension stiffness respectively, $x_{1}$ and $x_{2}$ are the tire displacement and body displacement with respect to their equilibrium positions respectively, $v_{1}$ and $v_{2}$ are the correspondingly speed of tire and body respectively, $a_{1}$ and $a_{2}$ are the correspondingly acceleration of tire and body respectively, $c_{e} q$ is the equivalent damping coefficient of HESA, $F$ is the maximum excitation force, $t$ is the time, and $\omega$ is the excitation frequency.

So the steady-state response of the relative motion between sprung mass and the unsprung mass would be:

$$
x_{2}-x_{1}=\left(X_{2}-X_{1}\right) \sin (\omega t-\theta)
$$

and

$$
X_{2}-X_{1}=\frac{m_{2} \omega^{2} F}{\sqrt{b_{1}+b_{2} c_{e} q^{2}}}
$$

where $b_{1}=\left[m_{1} m_{2} \omega^{4}+k_{1} k_{2}-\left(m_{1} k_{2}+m_{2} k_{1}+m_{2} k_{2}\right) \omega^{2}\right]^{2}$, $b_{2}=\left[k_{1}-\left(m_{1}+m_{2}\right) \omega^{2}\right]^{2} \omega^{2}$.

The energy-dissipative power under harmonic force excitation can be written as [16]:

$$
P_{\text {ave }}=\frac{c_{e q} \omega^{2}}{2}\left(X_{2}-X_{1}\right)^{2}
$$

Hence, the average power can be recast as:

$$
P_{\text {ave }}=\frac{b_{3} c_{e q}}{\left(b_{1}+b_{2} c_{e q}^{2}\right)}
$$

where $b_{3}=\frac{m_{2}^{2} \omega^{6} F^{2}}{2}$.

Assuming $c_{e}$ is the equivalent damping coefficient due to the electronic load, and $c_{c}$ is the equivalent damping coefficient of the hydraulic rectifier, the hydraulic motor, pipes and so on. The total damping of the system is given by

$$
c_{e q}=c_{c}+c_{e}
$$

Equation (6) shows the energy-dissipative power by damping. The recyclable power is $\frac{c_{e}}{c_{e q}} P_{a v e}$, and the part $\frac{c_{c}}{c_{e q}} P_{a v e}$ is the inevitable loss. In the circuit, the energy dissipated by internal resistance of the generator is also inevitable, so the energy-recyclable power delivering to the electrical load is given by

$$
P_{\text {har }}=\frac{R_{\text {load }}}{R_{\text {in }}+R_{\text {load }}} \frac{c_{e}}{c_{\text {eq }}} P_{\text {ave }}
$$

During the process of oil flow driving the hydraulic motor, the rotational speed and the torque can be written as:

$$
\begin{gathered}
n=\frac{Q}{q} \eta_{v} \\
T=\frac{\Delta P_{e} q}{2 \pi} \eta_{m}
\end{gathered}
$$

respectively, where $Q$ is the volumetric flow rate, $q$ is the motor displacement, $\eta_{v}$ and $\eta_{m}$ are the volumetric efficiency and the mechanical efficiency of the hydraulic motor respectively, and $\Delta P_{e}$ is the pressure drop of the hydraulic motor.

As the generator drove by the motor, the back electromotive force and torque is given by

$$
\begin{gathered}
V_{e}=k_{v} w \\
T=J \ddot{\theta}+k_{t} I
\end{gathered}
$$

respectively, where $k_{v}$ and $k_{t}$ are the back electromotive force coefficient and the torque constant respectively, $w$ and $\ddot{\theta}$ are the angular speed and acceleration of the rotor respectively, $J$ is the inertia of the rotor, and $I$ is the current.

It is noted that the inductance and the inertia of the rotor would not dissipate energy. In common with most previous investigations [17], inductance and the inertia of the rotor are ignored in this study, and the simplest form of electromechanical coupling, $V_{e}=I\left(R_{\text {in }}+R_{\text {load }}\right)$ is assumed in the circuit. Therefore, we can get the pressure drop of the motor from Equations (9) to (12) as:

$$
\Delta P_{e}=\left(\frac{2 \pi}{q}\right)^{2} \frac{\eta_{v}}{\eta_{m}} \frac{k_{v} k_{t}}{R_{\text {in }}+R_{\text {load }}} Q
$$

Assuming the equivalent sectional area based on the pressure drop of the motor is $A$, we can get the equivalent damping force $F=\Delta P_{e} A$, and the volumetric flow rate $Q=A v$. Hence, the equivalent damping coefficient of the motor can be obtained as:

$$
c_{e}=\left(\frac{2 \pi A}{q}\right)^{2} \frac{\eta_{v}}{\eta_{m}} \frac{k_{v} k_{t}}{R_{\text {in }}+R_{\text {load }}}
$$


Table 1: Parameters of the quarter car.

\begin{tabular}{lcc}
\hline Symbol & Valve & Units \\
\hline$m_{1}$ & 40 & $\mathrm{~kg}$ \\
$m_{2}$ & 350 & $\mathrm{~kg}$ \\
$k_{1}$ & 200 & $\mathrm{KN} / \mathrm{m}$ \\
$k_{2}$ & 20 & $\mathrm{KN} / \mathrm{m}$ \\
$F$ & 1000 & $\mathrm{~N}$ \\
$c_{c}$ & 520 & $\mathrm{~N} / \mathrm{m} / \mathrm{s}$ \\
$k_{v}$ & 0.07 & volts $/ \mathrm{rad} / \mathrm{s}$ \\
$k_{t}$ & 0.06 & $\mathrm{~N} / \mathrm{amp} / \mathrm{m}$ \\
$q$ & 5 & $\mathrm{cc} / \mathrm{cycle}^{2}$ \\
$A$ & 1963.5 & $\mathrm{~mm}^{2}$ \\
$\eta_{v}$ & 0.88 & \\
$\eta_{m}$ & 0.9 & \\
$R_{\text {in }}$ & 1 & ohm \\
\hline
\end{tabular}

3.1.1 Optimal load resistance based on maximum energy recovery

If substituting for the damping coefficient $c_{e}$ and $c_{e q}$ in Equation (8) from Equation (14) and Equation (7), and dividing both the numerator and denominator by $R_{\text {load }}$, the energy-recyclable power becomes as:

$P_{\text {har }}=\frac{b_{3} b_{4}}{\left(b_{1}+b_{2} c_{c}^{2}\right) R_{\text {load }}+d_{1}+\frac{d_{2}}{R_{\text {load }}}}$

where $\quad b_{4}=\left(\frac{2 \pi A}{q}\right)^{2} \frac{\eta_{v}}{\eta_{m}} k_{v} k_{t}$, $d_{1}=2\left(b_{1} R_{i n}+b_{2} b_{4} c_{c}+b_{2} R_{i n} c_{c}^{2}\right)$, $d_{2}=b_{1} R_{i n}^{2}+b_{2} b_{4}^{2}+2 b_{2} b_{4} c_{c} R_{i n}+b_{2} c_{c}^{2} R_{i n}^{2}$.

From Equation (15) we can get the optimal load resistance $R_{\text {load }}^{\text {opt }}$ for maximizing the energy-recyclable power is

$$
R_{\text {load }}^{\text {opt }}=\sqrt{R_{\text {in }}^{2}+\frac{b_{2} b_{4}^{2}+2 b_{2} b_{4} c_{c} R_{\text {in }}}{b_{1}+b_{2} c_{c}^{2}}}
$$

By substituting the optimal load resistance $R_{\text {load }}^{\text {opt }}$ to Equation (15), the maximum energy-recyclable power can be finally expressed as:

$$
P_{\text {har }}^{\max }=\frac{b_{3} b_{4}}{2 \sqrt{d_{1}\left(b_{1}+b_{2} c_{c}^{2}\right)+d_{2}}}
$$

To vividly express the relationship between load resistance and recyclable energy power, parameters of the quarter-car model are set as Table 1 lists.

Along with excitation frequency ranging from 0.3 to $30 \mathrm{~Hz}$, the dependence of energy-recyclable power on excitation frequency and load resistance can be illustrated in Figure 4. Figure 5 presents the corresponding contours, in which the black dotted line represents the optimal load resistance $R_{\text {load }}^{\text {opt }}$ at different excitation frequencies. There are two undamped natural frequencies of the dual-mass system:

$$
f_{1}=\frac{\sqrt{2}}{4 \pi} \sqrt{d_{3}+\sqrt{d_{3}^{2}-\frac{4 k_{1} k_{2}}{m_{1} m_{2}}}}
$$

$$
f_{1}=\frac{\sqrt{2}}{4 \pi} \sqrt{d_{3}-\sqrt{d_{3}^{2}-\frac{4 k_{1} k_{2}}{m_{1} m_{2}}}}
$$

where $d_{3}=\left(\frac{k_{1}+k_{2}}{m_{1}}+\frac{k_{2}}{m_{2}}\right)$.

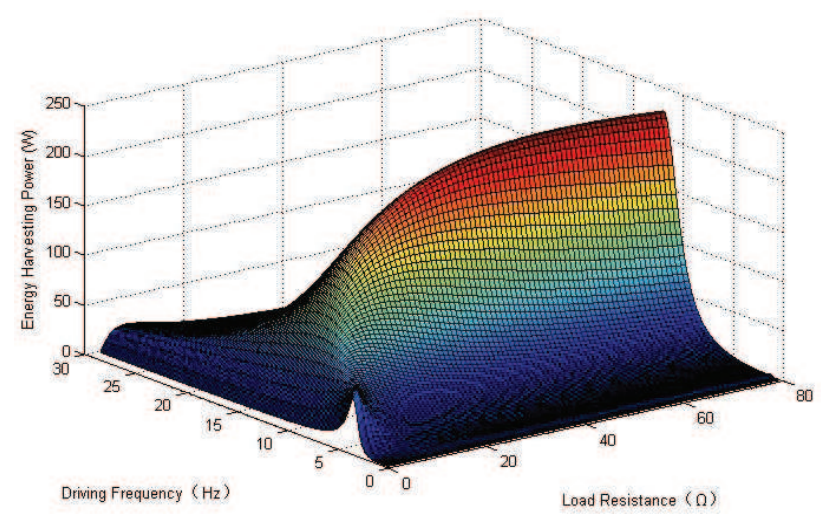

Fig. 4: Energy-recyclable power at different excitation frequencies and load resistance.

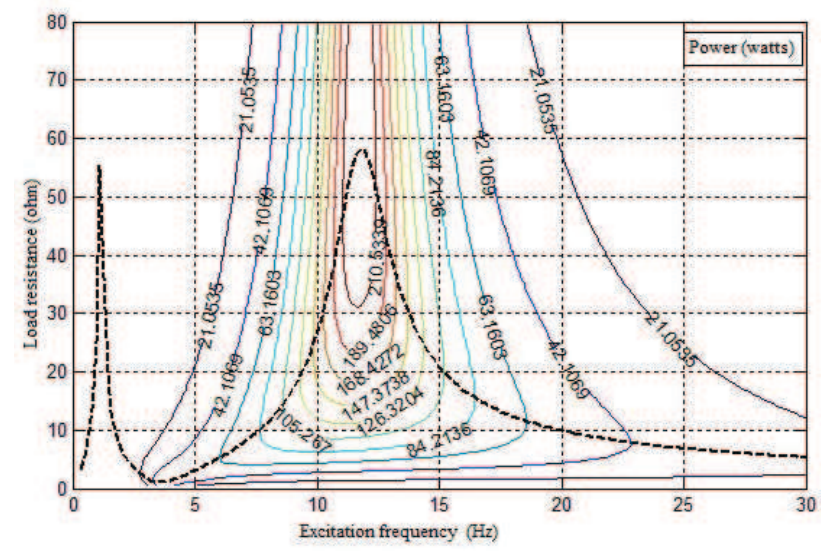

Fig. 5: Contours of energy-recyclable power.

By substituting the parameters with the values in Table 1 , we can get $f_{1}=1.15 \mathrm{~Hz}$ and $f_{2}=11.8 \mathrm{~Hz}$. If considering the dual-mass system as a whole, the undamped natural frequency of the system would be:

$$
f_{3}=\frac{1}{2 \pi} \sqrt{\frac{k_{1}}{m_{1}+m_{2}}}
$$

The optimal load resistance shown in Figure 5 proves that the optimal load resistance reaches the extreme value 
Table 2: Maximum energy-recyclable power at the natural excitation frequencies.

\begin{tabular}{ccc}
\hline $\begin{array}{c}\text { Excitation } \\
\text { frequencies } / \mathrm{Hz}\end{array}$ & $\begin{array}{c}\text { Optimal load } \\
\text { resistance/ohm }\end{array}$ & $\begin{array}{c}\text { Maximum energy- } \\
\text { recyclable power/watts }\end{array}$ \\
\hline$f_{1}=11.8$ & 58.37 & 232 \\
$f_{2}=1.15$ & 58.37 & $<1$ \\
$f_{3}=3.60$ & 1.23 & 63 \\
\hline
\end{tabular}

when excitation frequencies are the natural frequencies. Table 2 shows the values of the maximum energy-recyclable power at the natural excitation frequencies. Through numerical simulations and basic experiments, Suda and Shiiba [17] concluded that the harvest energy would drop as the load resistance decreased, while Okada and Harada [1] drew a conclusion that harvest energy would rise as the resistance decreased in high excitation frequency range. From Figs. 3 and 4 we can get that the maximum energy-recyclable power appears only when the load resistance is the optimal load resistance. It means that for any excitation frequency, the energy-recyclable power first increases and then decreases as the load resistance increases gradually, and the optimal load resistance is the critical value. Stephen [16] claimed that the maximum power $\left(R_{\text {load }}^{N G}\right)$ was delivered to the load resistance when its resistance is equal to the sum of the coil internal resistance $\left(R_{i n}\right)$ and the electrical analogue of the mechanical damping coefficient $\left(\frac{k_{v} k_{t}}{c_{c}}\right)$, which could be expressed as follows:

$$
R_{\text {load }}^{N G}=R_{\text {in }}+\frac{k_{v} k_{t}}{c_{c}}
$$

By substituting the parameters with the values in Table 1, we can get $R_{\text {load }}^{N G}=58.37 \mathrm{ohm}$ in Equation (21). Only when the excitation frequency is $f_{1}$ or $f_{2}$, the maximum power is delivered to the load resistance. In other frequencies, the maximum power delivered to the load resistance is lower than $R_{\text {load }}^{N G}$.

\subsubsection{Optimal damping ratio based on the maximum} energy-recyclable power

As to maximum energy-recyclable power, it's easier to analyze it by load resistance than by damping ratio of the suspension. But to the ride comfort, it's more convenient to evaluate it by damping ratio than by load resistance. Equations (7) and (14) we can get the damping ratio

$$
\xi=\frac{c_{c}+\frac{b_{4}}{R_{\text {in }}+R_{\text {load }}}}{2 \sqrt{k_{2} m_{2}}}
$$

If the damping ratio meets $\xi[0.1,0.5]$, from Equation (22) we can get that the electrical load would be $R_{\text {load }} \in[13,3259] \mathrm{ohm}$. Hence, when the optimal load resistance $R_{\text {load }}^{\text {opt }}<13 \mathrm{ohm}$, we define the optimal electrical load $R_{\text {load }}^{\text {opt }}=13 \mathrm{ohm}$ in order to ensure the ride comfort. Figure 6 shows the energy-recyclable power at different excitation frequencies and damping ratios, and Figure 7 shows the corresponding contours. According to Figures 6 and 7, it's obvious that there leaves little energy to be recovered when the excitation frequency is lower than 3 $\mathrm{Hz}$. And when the excitation frequency is higher than 18 $\mathrm{Hz}$, we can design larger damping ratio because it's better for both the driving safety and energy harvest.

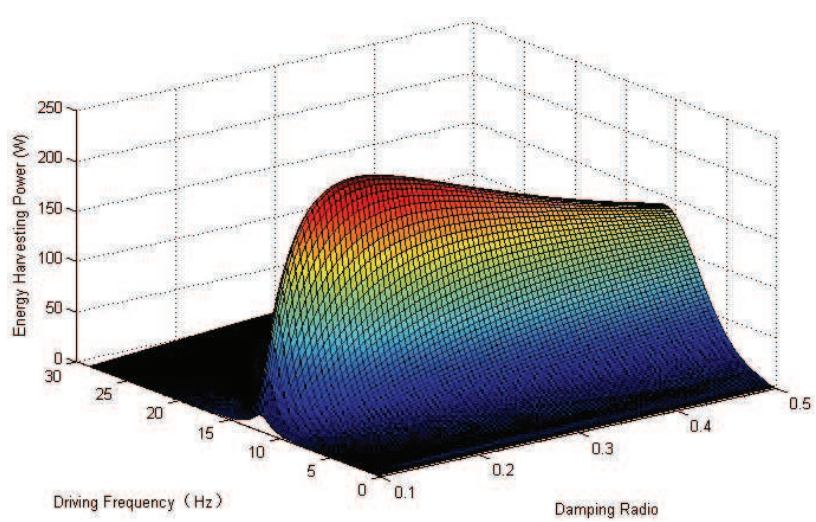

Fig. 6: Energy-recyclable power at different excitation frequencies and damping ratios.

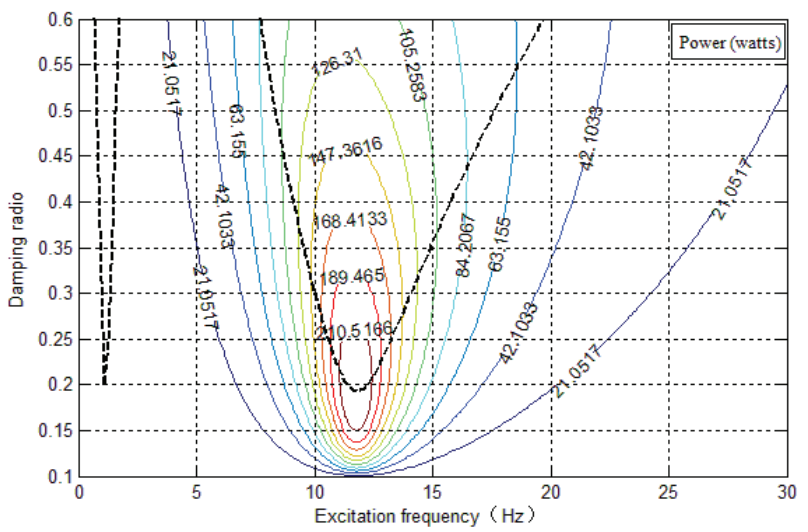

Fig. 7: Contours of energy-recyclable power.

What's more, from Figures (4-7), it's clear that: (1) the maximum energy-recyclable power appears when the damping ratio is 0.193 (good ride comfort) and the excitation frequency is $f_{1}$ (wheel resonant); (2) the energy-recyclable power is more sensitive to excitation frequency than to damping ratio. So this energy-regenerative scheme is more suitable for the car 
often subjected to an excitation frequency close to the wheel resonant frequency.

\subsection{Motion excitation}

Assuming the tire is subjected to harmonic displacement excitation, the dynamic equations can be written as:

$$
m_{2} a_{2}+c_{e q}\left(v_{2}-v_{1}\right)+k_{2}\left(x_{2}-x_{1}\right)=0
$$

and

$$
\begin{aligned}
m_{1} a_{1}+ & c_{e q}\left(v_{1}-v_{2}\right)+k_{2}\left(x_{1}-x_{2}\right) \\
& +k_{1}\left(x_{1}-X_{0} \sin \omega t\right)=0
\end{aligned}
$$

where $X_{0}$ is the maximum excitation amplitude.

Similarly, the energy-dissipative power can be expressed as Equation (7), and its energy-recyclable power, $P_{\text {har } 1}$ meets

$$
\frac{P_{\text {har } 1}}{P_{\text {har }}}=\left(\frac{X_{0} k_{1}}{F}\right)^{2}
$$

where the parameters $F, X_{0}$ and $k_{1}$ are all constant. It is obvious that the tire subjected to harmonic displacement excitation has the same characteristic as subjected to harmonic force excitation. Hence, we omit the analysis on harmonic displacement excitation.

\section{Active Control Exploration of HESA}

Schematic of the test bench is shown in Figure 1, and the generator is connected to electric load. The test bench is executed on HESA according to the standard of QC/T 545-1999, and the excitation frequency and amplitude is $1.667 \mathrm{~Hz}$ and $50 \mathrm{~mm}$ respectively in accordance with the standard. For the HESA trial prototype, the diameter of the inner chamber and piston rod is 50 and20 $\mathrm{mm}$ respectively, and the working volume of hydraulic motor is $11.0 \mathrm{cc} / \mathrm{rev}$.

By regulating the electric load to make the current zero in the circuit as shown in Figure 7, we can get that the rotational speed of the motor is about2820 rev/min, and the damping force is $F \in[-2000,2000] N$. Using theoretical calculations, it can be determined whether the speed of the motor is reasonable or not. Within one cycle, the total flow of the hydraulic cylinder is:

$$
V=\frac{100 \pi}{4} 50^{2}+\frac{100 \pi}{4}\left(50^{2}-20^{2}\right)=0.3613 L
$$

and the corresponding volume flow is:

$$
Q=V \times 1.667 \mathrm{~Hz}=36.13 \mathrm{~L} / \mathrm{min}
$$

The volumetric efficiency is approximate to 0.88 when the rotate-speed ranges from 2500 to $3000 \mathrm{rev} / \mathrm{min}$. So we can get the average speed of motor:

$$
n_{0}=\frac{Q}{11.0 \mathrm{cc} / \mathrm{rev}} \times 0.88=2890 \mathrm{rev} / \mathrm{min}
$$

Table 3: Stable motor speed and damping force at different current values.

\begin{tabular}{ccc}
\hline $\begin{array}{c}\text { Current } \\
\text { lamp }\end{array}$ & $\begin{array}{c}\text { Rotational speed } \\
\text { /rev/min }\end{array}$ & $\begin{array}{c}\text { Maximum Damping } \\
\text { force/N }\end{array}$ \\
\hline 0 & 2820 & {$[-2000,2000]$} \\
5 & 2510 & {$[-3600,3500]$} \\
10 & 2260 & {$[-5800,5700]$} \\
20 & 1900 & {$[-8800,8500]$} \\
\hline
\end{tabular}

Compared with theoretical average speed, the average speed we get from the experiment is about $2820 \mathrm{rev} / \mathrm{min}$, and the $2.4 \%$ deviation is acceptable.

By adjusting the electric load, the current can be stabilized to $5 \mathrm{amp}$, and the speed of the motor declines rapidly to about $2510 \mathrm{rev} / \mathrm{min}$ correspondingly. However, there is a break during the decreasing process of the rotational speed. That is to say, the speed drops suddenly and then returns to the stable speed quickly as shown in Figure 9. The reason of this phenomenon is that, when the current is changed to $5 \mathrm{amp}$ in a short time, the oil pressure rises rapidly and accumulator 1 absorbs some oil accordingly, which results in the reduction of oil flowing through the hydraulic motor and the decline of the rotational speed of the motor. When accumulator 1 reaches its equilibrium position, the rotational speed also reaches the balanced range. Along with this, the damping force increases as the pressure rises, and it is in the range of $[-3600,3500] N$ as shown in Figure 10. From Figure 10 , it is also noticed that there is a break during the damping force changed its direction. That is because the mounting clearance between the HESA prototype and the test bench isn't eliminated completely, and we can even hear the impact sound in high frequency excitation.

The current drops from 5 to $0 \mathrm{amp}$ some time later, and a speed break of the motor comes out again. The speed of the motor increases sharply to the stable rotate speed around $2820 \mathrm{rev} / \mathrm{min}$, and the damping force also returns to its initial range. This phenomenon is believed to result from pressure drop of the system, since accumulator 1 would release some oil lubricating the motor during the process. When the current rises to 10 or 20 amp suddenly, a break of the rotational speed appears again, and the break becomes more prominent if the current value changes more greatly. Table 3 shows the stable motor speed and the damping force at different current values. We can get from the test that the damping force can be changed by adjusting the circuit current, which means the active control of HESA can be achieved by adjusting the circuit current.

Actually, the control of the damping force of HESA is influenced by many factors, such as the size of hydraulic cylinder and accumulator 1, pre-load of accumulator 1 , parameters of the rectifier and so on, so some more work need to be done to achieve active suspension based on HESA. Firstly, hydraulic cylinder, accumulator 1 and hydraulic rectifier must match with reference vehicle. 


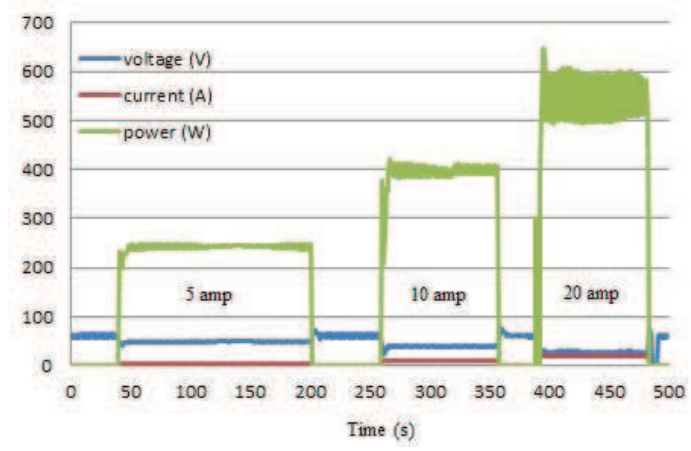

Fig. 8: Voltage and power at different current

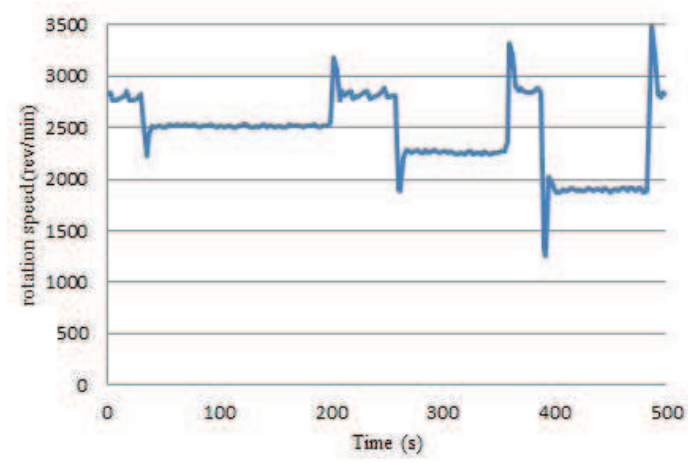

Fig. 9: Rotational speed of the motor.

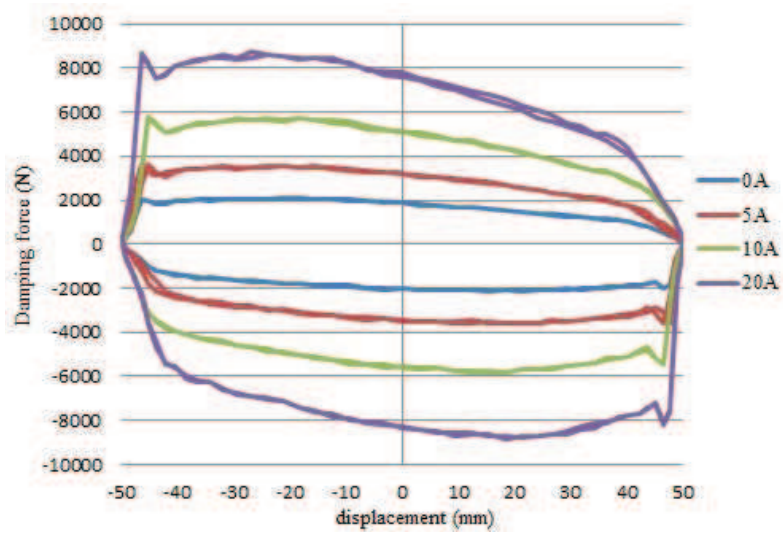

Fig. 10: Damping force.

Secondly, accumulator 1 should be matched with the road excitation to avoid dead zone during the active control of HESA. Finally, the relationship between the current and damping force should be clarified.

\section{Conclusions}

This work presents one kind of HESA, and optimal energy recovery analysis and active control of HESA are carried out based on a quarter-car model combined with simulation results. Based on the discussion above, the following conclusions can be drawn:

(1) For any excitation frequency, the energy-recyclable power first increases and then decreases as the load resistance increases gradually, and the optimal load resistance is changeable as the excitation frequency changes;

(2) The energy-recyclable power is more sensitive to excitation frequency than to damping ratio, and the requirements of ride comfort and safety can be met by changing the damping ratio properly without reducing energy-recyclable power obviously;

(3) The wheel resonant frequency is the ideal excitation frequency for suspension energy recovery system;

(4) The feasibility of active control of HESA is confirmed by the experiments, although there still need much further study.

\section{Acknowledgement}

The authors gratefully acknowledge the National Natural Science Foundation of China by 2011(Project No. 51075312) and Wanxiang Group Corporation Technology Center. Appreciation also applies to Dr. Bin Wang from DongFeng Automobile Co., LTD and Dr. Jun Yan from DongFeng Motor Corporation Technology Centre.

\section{References}

[1] Yohji Okada, Hideyuki Harada, Regenerative control of active vibration damper and suspension systems, Proceedings of the 35th IEEE Conference on Decision and Control, Kobe, Japan, 4715-4720 (1996).

[2] Yoshihiro Suda, Shigeyuki Nakadai and Kimihiko Nakano, Hybrid Suspension System with Skyhook Control and Energy Regeneration. Vehicle System Dynamics, 29, 619634 (1998).

[3] Abhijit Gupta, A. J. Jendrzejczyk, M. T.Mulcahy and R. J. Hull, Design of Electromagnetic Shock Absorbers. International Journal of Mechanics and Materials in Design, 3, 285-291 (2006).

[4] Kimihiko Nakano, Yoshihiro Suda, Combined Type SelfPowered Active Vibration Control of Truck Cabins, Vehicle System Dynamics, 41, 449-473 (2004).

[5] Yongchao Zhang, Fan Yu, Yonghui Gu, Xuechun Zheng, Isolation and Energy-regenerative Performance Experimental Verification of Automotive Electrical Suspension. Journal of Shanghai Jiaotong University, 42, 874-877 (2008). 
[6] Zhongjie Li, Zachary Brindak, and Lei Zuo. Modeling of an Electromagnetic Vibration Energy Harvester with Motion Magnification. Proceedings of the ASME 2011 International Mechanical Engineering Congress and Exposition, Denver, USA, November, 285-293 (2009).

[7] Yasuhiro Kawamoto, Yoshihiro Suda, Hirofumi Inoue, Takuhiro Kondo, Modeling of Electromagnetic Damping for Automobile Suspension, Journal of System Design and Dynamics, 1, 524-535 (2007).

[8] kun Huang, Fan Yu, Yongchao Zhang, Model Predictive Controller Design for Electromagnetic Active Suspension, Journal of Shanghai Jiaotong University, 44, 1619-1624 (2010).

[9] XuexunGuo, LinXu, Hydraulic Electromagnetic Energyregenerative Shock Absorber, Invention patent, (2010).

[10] LinXu, Structure Designs and Evaluation of Performance Simulation of Hydraulic Transmission Electromagnetic Energy-regenerative Active Suspension, SAE 2011 World Congress and Exhibition, Michigan, 0760 ( 2011).

[11] Zhigang Fang, XuexunGuo, LinXu, Energy Dissipation and Recovery of Vehicle Shock Absorbers, SAE Commercial Vehicle Engineering Congress, Illinois, 2037 (2012).

[12] Segel L., Xiaopei Lu, Vehicular resistance to motion as influenced by road roughness and highway alignment. Australian Road Research, 12, 211-222 (1982).

[13] A. Browne, J. Hamburg, On road measurement of the energy dissipated in automotive shock absorbers, Symposium on Simulation and Control of Ground Vehicles and Transportation Systems, Anaheim CA, USA, 80, 167-186 (1986).

[14] Lei Zuo, Peisheng Zhang, Energy Harvesting, Ride Comfort, and Road Handing of Regenerative Vehicle Suspensions, ASME 2011 Dynamic Systems and Control Conference, Oct 31-Nov 2, 2011, Arlington, Virginia, USA, 295-302 (2012).

[15] QC/T545-1999, Automobile Shock Absorber Bench test methods, Standards of Automotive Industry in PRC (1999).

[16] N. G. Stephen. On energy harvesting from ambient vibration. Journal of Sound and Vibration, 293, 409-425 (2006).

[17] Yoshihiro Suda, Taichi Shiiba, A New Hybrid Suspension System with Active Control and Energy Regeneration, Vehicle System Dynamics, 641-654 (1996).

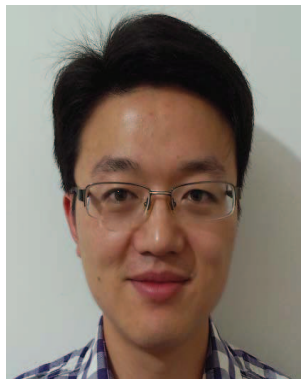

Zhigang

Fang was born in Huangshi, China in 1984. He received his $\mathrm{BS}$ and MS degree in vehicle engineering from Wuhan University of Technology, Wuhan, China, in 2008 and 2011 respectively. $\mathrm{He}$ carries out his $\mathrm{PhD}$ research in the area of energy recovery since 2010 in Wuhan University of Technology too.

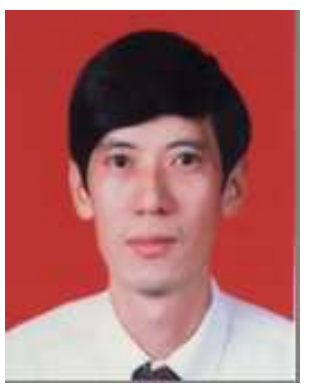

\section{Xuexun}

Guo is a professor in the School of Automotive Engineering in Wuhan University of Technology, a committee member of SAE-China SUV Branch, and a committee member of China Tractor Society. He does research in vehicle dynamics and control, simulation and control of transmission system and braking system, energy-regenerative shock absorber, hydraulic retarder and torque converter.

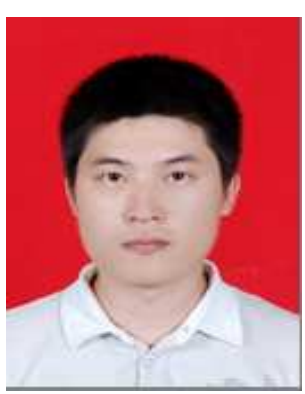

Lin

Xu received his B.Sc. and M.Sc. degree in vehicle engineering from Wuhan University of Technology, Wuhan, China, in 2002 and 2006 respectively. He was born in Jingzhou?China in 1979. He carried out his Ph.D. research in the area of energy recovery since 2007 in Wuhan University of Technology. He is now a teacher of Wuhan University of Technology and his research is electrohydraulic energy- regenerative type shock absorber for theoretical and experimental research.

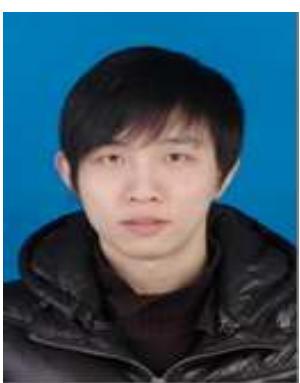

Han Zhang is a Ph.D. Candidate in Wuhan University of Technology, China. His research interests are in vehicle system dynamics, especially suspension system dynamic analysis and vehicle test technology. 